\title{
Analysis of ways to control the process of vibration machining in order to increase its efficiency and reduce the technological cost of processed parts
}

\author{
Galina Prokopetz ${ }^{1}$, Anna Azarova ${ }^{1, *}$, and Anatoliy Prokopetz ${ }^{1}$ \\ ${ }^{1}$ Don State Technical University, 1, Gagarin sq., 344003, Rostov-on-Don, Russia
}

\begin{abstract}
The article analyzes the features of vibration processing in the framework of the task of managing the efficiency of the process and reducing its technological cost, outlines the main ways to solve this problem, and provides examples of automatic control schemes to control the process by quality parameters and to stabilize the process.
\end{abstract}

\section{Introduction}

Mechanical processing of mechanical engineering parts involves changing the shape, size and quality indicators of the surface and the surface layer of the parts by mechanical action using the appropriate tool. Depending on the effect of processing on the shape and size of parts, there are dimensional and dimensionless processing of parts.

Vibration machining of parts belongs to the class of dimensionless machining methods. It occurs because, in vibration processing, the change in shape and size of the parts varies within the range of roughness of the workpiece. That is, in the case of small high-precision parts, as well as low-rigid parts of small size, it is unacceptably to neglect the change in the size or shape of the surfaces, as well as their mutual location. Also, when removing the burrs and rounding off the sharp edges one must maintain certain dimensions during machining. For the rest, the impact of the working medium particle on the processed surface is aimed at formation of the surface quality and surface layer indicators: roughness, microhardness, residual stresses in the thin surface layer, gloss, etc. As a result, the specified performance parameters are provided: wear resistance, corrosion resistance, fatigue strength, reflectivity, etc. Thus, vibration processing has a wide technological capability.

In connection with the above vibration processing is applied widely and can be used at various stages of parts' manufacturing, starting with the procurement process, ending with finishing operations performed with high surface purity or coating. The main tasks that can be solved with the help of vibration processing include the following:

- cleaning of workpieces from residues of molding mixtures;

- deburring after machining;

- preparing the surface of parts for coatings and applying certain types of coatings [1,2];

*Corresponding author: azarovaai@mail.ru 
- finishing grinding or polishing the surfaces of details [3, 4];

- hardening of the surface of details by increasing the microhardness of the surface, creating compressive residual stresses, stabilizing residual stresses [15], etc.

Vibration processing is based on the application of low-frequency vibrations with characteristics that ensure the mutual movement of the working medium and the processed parts for the achievement of specified indicators of the surface quality of the part.

Vibration units can have different designs and configuration, for example, be singlechamber or multi-chamber. Working chamber form can be V-shaped, toroidal, etc.

The formation of a predetermined shape of the working chamber loading mass trajectory, which includes the working medium, the processed parts and the process fluid, is provided mainly by the vibration parameters and the shape of the working chamber.

The working chamber (or working chambers for multi-chamber installations) is installed on elastic elements (elastic suspension), which allows it to oscillate in three coordinate directions, as well as to slightly rotate around horizontal axes. The magnitude of these rotations depends on the rigidity of the suspension and the unbalance of the vibrating part of the process system and has little effect on the shape of the mass circulation of the load, so they can be neglected in the vast majority of cases. Amplitude of oscillations is determined mainly by mass of unbalanced weights and angle between them. The frequency of oscillation is provided directly by a drive, which in most cases includes a motor and a multirack belt gear, or a motor with continuously variable output shaft speed control is used. A simplified diagram of a two-chamber installation for vibration processing is shown in Fig. 1.

Vibration processing is very specific. It is characterized by high complexity in describing the processes of forming quality indicators of processed parts. This is primarily due to the high flexibility of the tool (working environment), which in the process of processing in its mass repeats the shape of the part, which is a great advantage of this processing method, since it provides high uniformity of processing, without requiring the creation of complex kinematic relationships. But at the same time, each individual particle of the working medium when it collides with the surface of the workpiece has its own energy characteristics, which determine the useful work spent on separating the micro-chips (during abrasive processing), the depth and degree of plastic deformation (during hardening processing), adhesive bonds (when applying coatings), and others. Therefore, a large stochastic component is present as a result of processing. At the same time, the use of vibration processing primarily at the final stages of products' processing imposes special requirements for the stability of such operations and, as a result, requires reducing the influence of random factors on the result of processing.

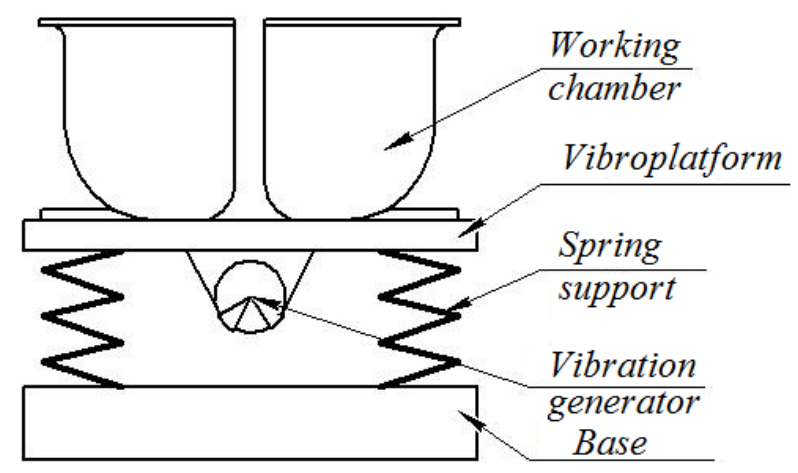

Fig. 1. A simplified diagram of the vibration processing unit. 
There is also a deterministic component in the error of quality indicators of the parts. Since the duration of the vibration treatment is quite long (but this disadvantage is compensated by the fact that an entire batch of parts is often processed simultaneously), there is wear of the working environment (mainly during abrasive processing). Wear of the working medium leads to changes in the cutting characteristics (or impact), which, accordingly, causes an increase in the scattering field of quality indicators.

One of the most important characteristics of the process operation is its profitability, which is reflected in the corresponding component of the technological cost of the part. Process cost depends on many factors, including labor intensity of part processing, tool and process fluid costs, equipment cost, etc. By analyzing the composition of these factors, it becomes clear that the technological cost of manufacturing a product depends largely on the reliability of the manufacturing process, and the stability of this process is one of the components of its reliability. The reliability of the vibration processing process affects, among other things, the complexity of the operation, the time between adjustments, the amount of depreciation and other factors that affect the technological cost of processing.

An important feature of vibration processing is the presence of a sufficiently large amount of tools for managing both the quality of processing and its efficiency, including the technological cost [5]. For their identification it is necessary to make analysis and sorting of factors during processing as well as possibilities of process control entity and the degree of influence on process stability and process costs operation vibration treatment.

Reducing the influence of the stochastic component of the process can be done in many ways, including:

- making changes to the design of elements of the technological system;

- using the additional sources of energy [6];

- optimization of technological parameters of processing;

- development of new tool (working) environments [7, 8, 9];

- reduction (or almost complete exclusion) of the influence of the human factor on the result of processing [10], including:

- mechanization of process elements;

- partial or full automation of the process;

- use of adaptive control systems, etc.

The results of the analysis of the technological operation of vibration processing to identify and specify the main ways to improve the reliability and efficiency of the process are summarized in table. 1 .

Almost all of these ways require some additional capital investment, as well as a set of experimental studies, some of which are already carried out [11, 12, 13, 14]. All this on the first stage will inevitably cause an increase in the technological cost of the processed products. However, the implementation of these options finally leads both to an increase in the quality of processed products and to a decrease in the technological cost at the subsequent stages due to:

- reducing the number of defects;

- reducing the complexity of processing;

- reducing of tool wear;

- reducing of costs for cleaning and recovery (or replacement) of technological fluid.

Besides, reducing the complexity of processing reduces the wear of the working environment, depreciation charges, energy costs, process fluid, wages, etc. It is typical (but not necessary) for vibration processing, that the processing of parts is carried out in batches at the same time (parallel processing). Moreover, it is possible to process different parts at the same time, if they are similar in size, material and requirements for the result of processing. When certain changes are made to the design of the working chamber, it becomes possible to process parts that differ significantly in size. Taking into account the 
above and the fact that the payback period of capital investments depends, among other things, on the size of the batch of processed workpieces, the implementation of a number of identified methods to increase the efficiency of the process looks very promising.

Table 1. Analysis of ways to control the technological operation of vibration processing in order to improve the quality of processing and reduce its technological cost price.

\begin{tabular}{|c|c|c|}
\hline $\begin{array}{c}\text { Management } \\
\text { method }\end{array}$ & Implementation & Implementation execution \\
\hline \multirow{6}{*}{$\begin{array}{l}\text { Changing the } \\
\text { design parameters } \\
\text { of the main } \\
\text { elements of the } \\
\text { technological } \\
\text { system }\end{array}$} & \multirow{2}{*}{$\begin{array}{l}\text { Changing the shape of the } \\
\text { load mass circulation flow }\end{array}$} & Changing the form of a working chamber \\
\hline & & $\begin{array}{l}\text { Inclusion of elements in the design for changing the } \\
\text { law of motion of the load mass }\end{array}$ \\
\hline & \multirow{2}{*}{$\begin{array}{l}\text { Changing the oscillation } \\
\text { characteristics of the } \\
\text { working chamber }\end{array}$} & $\begin{array}{l}\text { Changing the elastic properties of individual suspension } \\
\text { elements of the vibration platform }\end{array}$ \\
\hline & & Changing the design of the vibration generator \\
\hline & \multirow{2}{*}{$\begin{array}{l}\text { Introduction of additional } \\
\text { design elements }\end{array}$} & Dividing the working chamber into sections \\
\hline & & $\begin{array}{l}\text { Inclusion of elements in the design for fixing or } \\
\text { providing a certain law of movement of the processed } \\
\text { work pieces }\end{array}$ \\
\hline \multirow{4}{*}{$\begin{array}{l}\text { Modification of the } \\
\text { physical effects in } \\
\text { the processing of }\end{array}$} & \multirow{4}{*}{$\begin{array}{l}\text { The use of additional energy } \\
\text { sources }\end{array}$} & The imposition of ultrasound \\
\hline & & The imposition of the magnetic field \\
\hline & & The imposition of additional vibrations \\
\hline & & The use of chemical energy \\
\hline \multirow[t]{8}{*}{$\begin{array}{l}\text { Mechanization and } \\
\text { automation of } \\
\text { processing }\end{array}$} & $\begin{array}{l}\text { Mechanization of the } \\
\text { process of loading and } \\
\text { unloading parts }\end{array}$ & $\begin{array}{l}\text { Separation system using technological vibrations of the } \\
\text { working chamber }\end{array}$ \\
\hline & \multirow[t]{2}{*}{$\begin{array}{l}\text { Automating the process of } \\
\text { loading and unloading parts }\end{array}$} & $\begin{array}{l}\text { Separation system using technological vibrations of the } \\
\text { vibration platform }\end{array}$ \\
\hline & & Separation system using technological vibrations of the \\
\hline & \multirow[t]{2}{*}{ Control automation } & $\begin{array}{l}\text { vibration platform and elements of automatic quality } \\
\text { control of parts }\end{array}$ \\
\hline & & $\begin{array}{l}\text { Automatic quality control system for parts (outside the } \\
\text { separation system) }\end{array}$ \\
\hline & \multirow[t]{3}{*}{$\begin{array}{l}\text { Automation of the } \\
\text { processing cycle }\end{array}$} & $\begin{array}{l}\text { Automatic control system based on automatic control } \\
\text { systems for technological parameters }\end{array}$ \\
\hline & & $\begin{array}{l}\text { Adaptive control system for technological parameters } \\
\text { of the operation based on the feedback principle }\end{array}$ \\
\hline & & $\begin{array}{l}\text { Adaptive control system for technological parameters } \\
\text { of the operation based on an automatic control system } \\
\text { for quality indicators of parts, based on the feedback } \\
\text { principle }\end{array}$ \\
\hline \multirow{11}{*}{$\begin{array}{l}\text { Processing } \\
\text { optimization } \\
\text { according to the } \\
\text { parameters of the } \\
\text { technological cost }\end{array}$} & $\begin{array}{l}\text { Optimization of the load } \\
\text { mass composition }\end{array}$ & $\begin{array}{l}\text { The change of the ratio of the manufacturing } \\
\text { environment, intermediates and process fluid }\end{array}$ \\
\hline & \multirow{2}{*}{$\begin{array}{l}\text { Optimization of parameters } \\
\text { of the manufacturing } \\
\text { environment }\end{array}$} & Changing the composition of the process fluid \\
\hline & & $\begin{array}{l}\text { Changing the size characteristics of the working } \\
\text { medium particles }\end{array}$ \\
\hline & \multirow[t]{3}{*}{$\begin{array}{l}\text { Optimization of processing } \\
\text { modes }\end{array}$} & $\begin{array}{l}\text { Changing the amplitude of vibrations of the working } \\
\text { chamber }\end{array}$ \\
\hline & & $\begin{array}{l}\text { Changing the oscillation frequency of the working } \\
\text { chamber }\end{array}$ \\
\hline & & $\begin{array}{l}\text { Changing the washing speed of the loading mass by } \\
\text { process fluid }\end{array}$ \\
\hline & \multirow[t]{3}{*}{$\begin{array}{l}\text { Optimization of operation's } \\
\text { structure }\end{array}$} & $\begin{array}{l}\text { Variation of working chamber oscillation frequency } \\
\text { within one technological operation }\end{array}$ \\
\hline & & $\begin{array}{l}\text { Variation of working chamber oscillation amplitude } \\
\text { within one technological operation }\end{array}$ \\
\hline & & $\begin{array}{l}\text { Customization of operation's structure for a typical } \\
\text { part's processing }\end{array}$ \\
\hline & \multirow{2}{*}{$\begin{array}{l}\text { Optimization of the } \\
\text { processing cycle's structure }\end{array}$} & Multi-stage processing within a single operation \\
\hline & & Multi-stage processing with splitting into operations \\
\hline
\end{tabular}


The list of methods to reduce the labor input and technological cost of the vibration treatment operation is far from complete. However, they are either partially implemented or largely justified theoretically.

Let us consider three options:

1. Separation system using technological vibrations of the vibration platform and elements of automatic quality control of parts.

2. Adaptive control system for technological parameters of the operation based on the feedback principle.

3. Use of multi-stage processing.

\section{Option 1}

Vibration processing of parts has a number of specific features. For example, the presence of a vibrating working chamber, on the one hand, complicates the process of replacing a batch of processed parts with a batch of blanks without stopping the process, on the other hand, it allows to automate the process of unloading parts by using the already existing directional technological vibrations (vibrotransportation). However, this requires the design of special devices. At the same time, by including a tool for automatic control in such a device, you can accurately determine the moment when the operation ends and thus minimize its labor intensity. Fig. 2 presents a block diagram of the automatic control module for such control when processing parts in the free loading state.

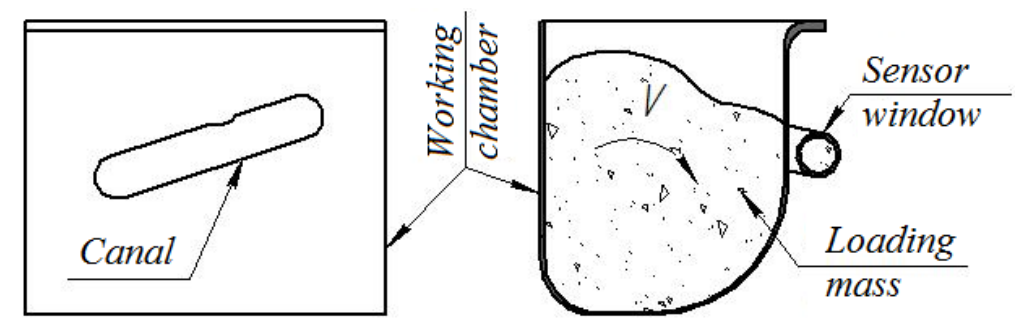

Fig. 2. Simplified automatic control scheme for controlling the process of vibration processing according to the quality parameters of the processed parts when processing parts in the free loading state.

The presence of a vibrating working chamber, on the one hand, complicates the process of replacing a batch of processed parts with a batch of blank ones without stopping the process. On the other hand, it allows to automate the process of unloading parts by using the already existing directional technological vibrations (vibrotransportation).However, this requires the design of special devices. At the same time, by including a tool for automatic control in such a device, you can accurately determine the moment when the operation ends and thus minimize its labor intensity. Fig. 2 presents a block diagram of the automatic control module for such control when processing parts in the free loading state.

This system is based on the vibrotransportation effect. In the working chamber, two holes are made in which valves are installed with dimensions that correlate with the dimensions of the parts. Under the pressure of the load mass flow, a certain amount of working medium with parts enters the holes. When passing through the channel, the parts pass under the sensor window, which captures the roughness of their surface and compares it with the specified value. If the specified number of parts has the required roughness, the corresponding signal is given and the processing stops or proceeds to the next stage, for example, separation. This means extraction of the processed parts without stopping the 
process system. So the minimum time for performing a technological operation can be ensured and the technological cost of processing parts can be accordingly reduced.

Such a control scheme can also be used to determine the moment of corrective adjustment according to the criterion of critical dimensional wear of the working medium. When the average size of the working medium particle reaches the minimum permissible value, a signal of its necessary replacement should be sent. Thus the working medium will fully run out its resource.

\section{Option 2}

If the main task is to control the technological parameters of the process, then the appropriate sensor can be used to monitor the wear of the working medium particles, for example, by changing their size or weight. Based on the results of such monitoring, the moment of adjustment of the technological system and replacement of the working environment can be determined. Similarly, the stability of the circulation rate can be controlled, which can be used as a complex controlled parameter to ensure the stability of the quality indicators of the processed parts. An example of automatic control of the load mass circulation rate is shown in Fig. 2.

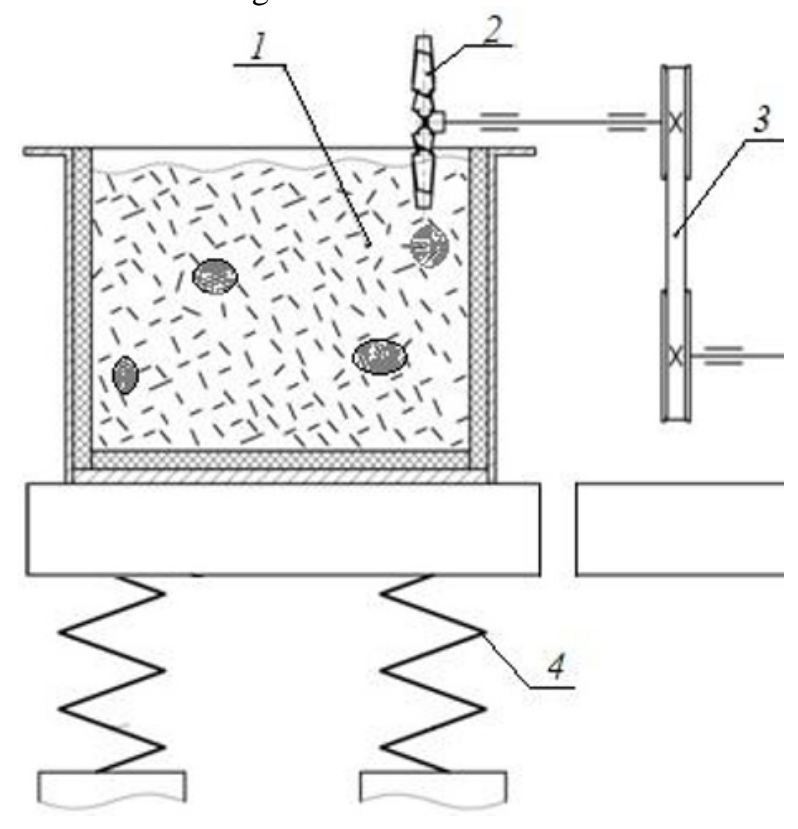

Fig. 3. Schematic diagram of the automatic control mechanism in the system of adaptive control of the process of vibration processing of parts: 1-working chamber with the load mass; 2 -mechanical sensor for measuring the speed of circulation of the load mass; 3-mechanical transmission; 4-elastic suspension.

Obtaining information about the change in the velocity of circulation is carried out using a mechanical gauge partly immersed in the moving mass load. When the circulation rate changes, the information is transmitted to the comparing device, where a control signal is generated to change the corresponding technological parameter (for example, the amplitude). Its changing leads to the return of the load mass circulation rate to the originally set value, i.e. the stabilization takes place. And the stabilization of the technological process of vibration processing creates prerequisites for its optimization, for example, in terms of labor input or technological cost. 


\section{Option 3}

While solving complex technological problems of finishing processing, when it is necessary to obtain high quality of the part's processed surface, especially in case of high initial roughness, multi-stage vibration processing of parts by free abrasives is used. Vibroprocessing makes it possible in some cases to significantly reduce the part's processing time, i.e. to increase its efficiency, to ensure the required accuracy and quality of parts and to improve their performance. In many cases, multistep processing is the only way to obtain a given surface roughness

Unlike dimension processing, where the concept of size tolerance is widely used, vibroprocessing with free abrasives, which as previously mentioned, is generally dimensionless. Therefore the concept of surface quality tolerance here is conditional. One of the main quality indicators that are generated by vibration processing is the surface roughness of the part. Requirements to roughness parameters are established on the basis of analysis of operational parameters of products, at that values of these parameters can be calculated both from theoretical and empirical dependencies.

When designing a process of multi-stage vibration treatment with free abrasives, it is necessary not only to set nominal values of initial and specified surface roughness for each stage of surface treatment of the part, but also to assign a tolerance for each stage.

At present, several techniques have been developed for designing multi-stage processes of vibration-abrasive finishing of parts. One of them is based on the application of the method of sequential refinements. The values of the refinements used in this procedure and obtained as a result of a large volume of experimental studies are given in Table 2.

Table 2. Values of refinement coefficients during treatment with free abrasives.

\begin{tabular}{|c|c|c|}
\hline Processing method & Processing type & Size of refinement coefficient $\boldsymbol{\varepsilon}$ \\
\hline Vibroprocessing & draft & $1.5-2$ \\
& semi-fair & $3-6$ \\
& fair & $2-3$ \\
\hline
\end{tabular}

Based both on the initial (typically obtained by forming processing) and the desired surface quality index of the part, the vibration cycle is split into steps. A process system is selected for each step that can provide a predetermined surface quality index of the part. It is connected with the fact that each technological system has the technological potential and means processing of details with certain minimum and maximum sizes of details, it is characterized by a certain coefficient of specification $\varepsilon$, etc. For the organization of process the technologist has to define quantity of sequentially working technological systems. This process can be represented by the following sequence:

1. The technological system which provides achievement of the set indicator of roughness of a surface is chosen, $\omega R_{a \text { part }} \leq \mathrm{TR}_{\mathrm{a}}{ }^{\text {set }}$ (where $\omega \mathrm{R}_{\mathrm{a} \text { part }}$ - the dispersion field of roughness of a surface of a detail after performance of the last step of processing, $\mathrm{TR}_{\mathrm{a}}{ }^{\mathrm{set}}$ the set size of parameter's dispersion). The technological system selected in this algorythm will work at the last stage, i.e. has the number $\mathrm{N}$.

2. Then, based on the dependence $\omega \mathrm{R}_{\mathrm{a}}{ }^{\text {init } \mathrm{N}}=\mathrm{TR}_{\mathrm{a}}{ }^{\text {set }} \varepsilon_{\mathrm{TC}}$ the value of initial roughness of a surface for $\mathrm{N}$ step of processing is obtained. This roughness at the same time is result of processing on step $\mathrm{N}-1$, i.e. $\omega \mathrm{R}_{\mathrm{a}}^{\text {init }} \mathrm{N}=\omega \mathrm{R}_{\mathrm{a}}{ }^{\text {set } \mathrm{N}-1}$. Based on this, the corresponding $\mathrm{N}-1$ technological system is selected.

3. Going further, the approximate value of roughness of a surface of a detail, being conditionally initial for N-1 step, and reached at N-2 step is calculated: $\omega R_{a}{ }^{\text {part N-2 }}=\omega R_{a}^{\text {init N- }}$ ${ }^{1}=\mathrm{TR}_{\mathrm{a}}{ }^{\mathrm{N}-1} \varepsilon_{\mathrm{TC}}{ }^{\mathrm{N}-1}$. If $\omega \mathrm{R}_{\mathrm{a}}{ }^{\mathrm{init}-1} \geq \omega \mathrm{R}_{\mathrm{a}}{ }^{\text {init }}$, then the whole process of achievement of roughness of a part's surface can be realized by two consistently working technological systems, i.e. 
$\mathrm{N}=2$. If the condition is not met, another technological system is selected, and so on until the condition is reached.

\section{Conclusion}

Vibration processing of mechanical engineering parts is a very promising and fairly universal method of processing. Wide technological capabilities of vibration processing during processing of parts of different configuration, made of different materials, it's high technical and economic indicators, as well as capabilities of mechanization and automation allow it to occupy an important place in finishing operations. However, many of its technological capabilities and efficiency gains have yet to be disclosed. This requires indepth analysis, systematization and specification of the main ways to improve the reliability of the vibration processing as the main way to increase its efficiency and competitiveness.

\section{References}

1. V. Ivanov, V. Lebedev, I. Panahin, Journal of Friction and Wear 4, 339-342 (2014)

2. S. Shtyn, I. Davydova, Hardening technologies and coatings 8(128), 34-39 (2015)

3. K. Hamouda, H. Bournine, M. Tamarkin, A. Babichev, D. Saidi, H. Amrou, Materials Science 52(2), 216-221 (2016) doi: 10.1007/s11003-016-9946-9

4. Y. Kopilov, Journal of Machinery Manufacture and Reliability 39(3), 276-281 (2010).

5. S. Shtyn, V. Lebedev, V. Ivanov, A. Kapustyanskiy, A. University, Vestnik Akademicheskii P.A. Solovyov 2(41), 117-122 (2017)

6. M. Tamarkin, E. Tishchenko, I. Viyalikov, Russian Engineering Research 35, 740-744 (2015)

7. A. Shishkina, V. Lebedev, M. Chaava, Bulletin of Tula State University, Technical science 5, 278-282 (2016)

8. A. Shishkina, V. Lebedev, M. Chaava, Bulletin of the Bryansk State Technical University 5(58), 42-49 (2017)

9. V. Butenko, V. Lebedev, I. Davidova, G. Serga, Proceedings of E3S Web of Conferences, 01040 (2019)

10. A. Suslov, D. Petreshin, Russian Engineering Research 4, 418 (2015).

11. M. Tamarkin, E. Tishchenko, Russian Engineering Research 38(9), 726-727 (2018) doi: 10.3103/S1068798X18090277

12. A. Shishkina, V. Lebedev, E. Krupenia, Bulletin of the Rybinsk State Aviation Technological Academy named after P.A. Solovyov 2(41), 132-137 (2017)

13. M. Tamarkin, E. Tishchenko, A. Melnikov, E. Chernishov, Advances in Intelligent Systems and Computing 983, 661-669 (2019) doi: 10.1007/978-3-030-19868-8_64

14. V. Lebedev, A. Sokolov, I. Davidova, Strengthening technologies and coatings 142(158), 54-58 (2018) 